\title{
Haploinsufficiency of the schizophrenia and autism risk gene Cyfip1 causes abnormal postnatal hippocampal neurogenesis through microglial and Arp2/3 mediated actin dependent mechanisms
}

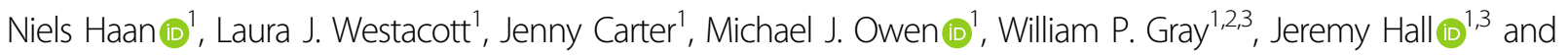 \\ Lawrence S. Wilkinson ${ }^{1,3,4}$
}

\begin{abstract}
Genetic risk factors can significantly increase chances of developing psychiatric disorders, but the underlying biological processes through which this risk is effected remain largely unknown. Here we show that haploinsufficiency of Cyfip1, a candidate risk gene present in the pathogenic 15q11.2(BP1-BP2) deletion may impact on psychopathology via abnormalities in cell survival and migration of newborn neurons during postnatal hippocampal neurogenesis. We demonstrate that haploinsufficiency of Cyfip 1 leads to increased numbers of adult-born hippocampal neurons due to reduced apoptosis, without altering proliferation. We show this is due to a cell autonomous failure of microglia to induce apoptosis through the secretion of the appropriate factors, a previously undescribed mechanism. Furthermore, we show an abnormal migration of adult-born neurons due to altered Arp2/3 mediated actin dynamics. Together, our findings throw new light on how the genetic risk candidate Cyfip 1 may influence the hippocampus, a brain region with strong evidence for involvement in psychopathology.
\end{abstract}

\section{Introduction}

Many psychiatric conditions show high heritability. Recent genetic studies in schizophrenia for example have identified up to 160 loci that increase risk for the disease $^{1,2}$ enriched within synaptic ${ }^{3}$, histone modifying and immune system genes ${ }^{4}$. CYFIP1 is a genetic risk factor for schizophrenia, autism and developmental delay, by virtue of its presence in the penetrant 15q11.2(BP1-BP2) copy number deletion ${ }^{5}$. Loss of one copy of this interval leads to substantially increased risk for disorder in both Wes$\operatorname{tern}^{6,7}$ and Han Chinese populations ${ }^{8}$. CYFIP1 haploinsufficiency is likely a major contributor to the $15 \mathrm{q} 11.2$

\footnotetext{
Correspondence: Niels Haan (HaanN@cardiff.ac.uk)

${ }^{1}$ Neuroscience and Mental Health Research Institute, Cardiff University, Hadyn Ellis Building, Maindy Road, Cardiff, UK

${ }^{2}$ Brain Repair and Intercranial Neurotherapeutics Unit, Cardiff University, Hadyn Ellis Building, Maindy Road, Cardiff, UK

Full list of author information is available at the end of the article
}

(BP1-BP2) psychiatric phenotype due to evidence of CYFIP1's involvement in a range of synaptic functions, including key roles in dendritic spine morphology and branching ${ }^{9-11}$. CYFIP1 protein influences synaptic function in two main ways. CYFIP1 interacts with fragile $\mathrm{X}$ mental retardation 1 (FMRP), the protein gene product of FMR1, to suppress translation of up to 800 target transcripts ${ }^{12}$. Secondly, it interacts with Wiskott-Aldrich syndrome protein family member 1 (WAVE1), a key mediator of cytoskeleton dynamics, to modulate ARP2/3 dependant actin branching ${ }^{13}$.

In the present work we focus on microglia and adult hippocampal neurogenesis (AHN) as important sites of CYFIP1 action and as potential contributors to psychiatric phenotypes arising from 15q11.2(BP1-BP2) copy number deletion. Adult-born hippocampal neurons, up to 700 of which are estimated to be born per day in humans ${ }^{14}$, develop through a well-defined series of 
cellular events, starting from proliferative stem cells through intermediate progenitors, migratory neuroblasts and immature neurons, before eventually forming mature granule cells, which distribute through the dentate gyrus $^{15}$. In rodent models, adult-born hippocampal neurons have been shown to be involved in a number of behavioural processes, spanning pattern separation ${ }^{16,17}$, spatial navigation ${ }^{18,19}$, memory turnover ${ }^{20}$, and acquisition $^{21}$ and extinction ${ }^{22}$ of fear memory. There behaviours are affected in psychiatric disorders including schizophrenia $^{23-26}$. Antipsychotic drugs, specifically atypical antipsychotics, also impact $\mathrm{AHN}^{27}$ and importantly, a number of functionally diverse schizophrenia risk genes, including DISC1 $^{28}, \quad$ SREB2/GPR85 ${ }^{29}$, CACNA1C $^{30,31}$ $D G C R 8^{32}$ and $m i R 137^{33}$, converge in their ability to modify AHN. Additionally, post-mortem findings from schizophrenia patients reveal changes in cell proliferation and expression of immature neuronal markers in the hippocampus $^{34-36}$.

Immune changes are another common hallmark of several psychiatric disorders, including schizophrenia, where evidence from epidemiological data ${ }^{37,38}$ and findings showing increased inflammatory factors in the circulation $^{39}$, and in post-mortem brain ${ }^{40}$ of schizophrenia patients, implicate altered immune function in the development of disease symptoms. Mechanistic insights linking immune changes to risk for psychopathology are however largely lacking. Recent interest has focused on microglia, the resident macrophage-like immune cells of the central nervous system, with data suggesting possible alterations in microglial functioning in both those at high risk and those diagnosed with schizophrenia ${ }^{41-44}$. The causal mechanism(s) by which microglia might contribute to pathogenesis remain unclear, though there is increasing evidence that, in addition to canonical immune functions, microglia also modify neuronal networks through synaptic pruning during development ${ }^{45}$ and into adulthood ${ }^{46}$. This microglial-mediated pruning is important for normal brain and behavioural functioning ${ }^{47}$. Additionally, microglia can modulate neuronal firing through direct cell-cell contacts ${ }^{48}$, and have been shown to be capable of phagocytosing both developing ${ }^{49}$ and adult ${ }^{50}$ neurons.

In this work we used a translationally relevant haploinsufficient mouse model to reveal hitherto undescribed effects of manipulating Cyfip1 on the survival and migration of newborn neurons in dentate gyrus hippocampus, key cellular processes in the development and function of the adult hippocampus. We also reveal the mechanisms mediating these effects, highlighting CYFIP1 effects on microglia mediated apoptosis and Arp $2 / 3$ dependent actin dynamics, respectively. We suggest these findings may have relevance to how Cyfip 1 haploinsufficiency can influence hippocampal function in the context of the markedly increased risk for psychiatric disorder arising from the $15 \mathrm{q} 11.2(\mathrm{BP} 1-\mathrm{BP} 2)$ copy number deletion.

\section{Materials and methods}

For full Materials and methods, please see Supplementary Information.

\section{Cyfip1 heterozygous knockout mice}

Cyfip $1^{\text {tm2a(EUCOMM)Wtsi }}$ animals (MGI:5002986, referred to hereafter as $C y f i p 1^{+-}$) were maintained heterozygously on a C57/BL6) background, for genotyping examples, see Fig. S1. Animals used were of mixed sex and 8-12 weeks old. Cyfip1 $1^{+/-}$and wild-type littermates were kept in conventional cages with $12 \mathrm{~h}$ light-dark cycle and ad libitum access to water and food at all time. All experiments were performed in accordance with the Animal (Scientific Procedures) Act 1986, and approved by the local animal welfare committee.

\section{Tissue harvesting and immunohistochemistry}

Paraformaldehyde fixed brains from 8 to 12-week-old Cyfip $1^{+1-}$ and wild-type littermates were prepared as previously described ${ }^{51}$. Free floating $40 \mu \mathrm{m}$ cryostat sections were used for immunohistochemistry. Immunohistochemistry was performed as previously described ${ }^{51}$, with a 1:12 stereotactic sampling rate. Detection of cleaved-caspase 3 used a modified protocol, where primary antibodies were incubated for $48 \mathrm{~h}$ in blocking solution at $4{ }^{\circ} \mathrm{C}$. Primary and secondary antibodies are described in Table S1.

\section{Primary hippocampal progenitor culture}

Hippocampal progenitors were isolated from P7 to P8 animals. Isolated hippocampi were dissociated in papain and progenitors were enriched through Optiprep density gradient centrifugation. Cells were cultured on poly-Llysine and laminin in the presence of EGF and FGF2.

\section{Primary microglial culture}

Microglia were isolated from P7 to P8 whole brain mixed glia through the shake-off method.

\section{Immunocytochemistry}

Fixed cultures were blocked and permeabilized with 5\% donkey serum and $0.1 \%$ Triton X100 in PBS (PBST) for $30 \mathrm{~min}$ at room temperature. Primary antibodies were applied in the same solution overnight at $4{ }^{\circ} \mathrm{C}$. Antibodies are detailed in Table S1. Following three washes in PBST, relevant secondary antibodies were applied in PBST for $2 \mathrm{~h}$ at room temperature. For identification of microglia, Alexa 568 conjugated IB4 (ThermoFisher I21412) was added to secondary antibody solutions at $2.5 \mu \mathrm{g} / \mathrm{ml}$. Cells were washed, counterstained with DAPI, and mounted for microscopy. 


\section{Conditioned medium}

Microglia conditioned medium was prepared by incubating $5 \times 10^{5}$ microglia per $\mathrm{ml}$ in progenitor medium for $24 \mathrm{~h} .25 \%$ conditioned medium was added to hippocampal cultures.

\section{Time-lapse imaging and cell tracking}

Primary hippocampal progenitors were plated on polyD-lysine and laminin coated 24 well plates, and cultured for 5 days. At 5 DIV, cultures were imaged on an inverted Leica DMI600B microscope, at $37^{\circ} \mathrm{C}$, with $5 \% \mathrm{CO}_{2}$ in air. Cells were imaged for $2 \mathrm{~h}$, with image acquisition every 5 min. Total distance covered was determined through manual cell tracking using the MTrackJ plugin in Image $^{52}$. A minimum of 50 cells ware analysed per condition per animal.

\section{Actin analysis}

Cells were fixed at 5 DIV with $4 \%$ paraformaldehyde in PBS, at $4{ }^{\circ} \mathrm{C}$ for $30 \mathrm{~min}$, permeabilised with $0.1 \%$ Triton X100 in PBS for $15 \mathrm{~min}$, and incubated with $300 \mathrm{nM}$ Alexafluor 488 conjugated DNAseI (ThermoFisher D12371) and $200 \mathrm{nM}$ Alexafluor 647 conjugated phalloidin (ThermoFisher A22287) for $30 \mathrm{~min}$. Fluorescence intensity was measured in ImageJ.

\section{Arp2/3 inhibition}

Cells were treated with $50 \mu \mathrm{M}$ CK-548 or $250 \mu \mathrm{M}$ CK666 in DMSO, or vehicle, for $2 \mathrm{~h}$ prior to time-lapse imaging. Drugs were present during imaging.

\section{Results}

Cyfip $1^{+/-}$animals have normal proliferation but increased numbers of adult-born immature neurons in the hippocampus

We first investigated cell proliferation in the dentate gyrus of the hippocampus. Animals received a single injection (i.p.) of $100 \mathrm{mg} / \mathrm{kg}$ BrdU $6 \mathrm{~h}$ prior to sacrifice. No significant differences in cells positive for BrdU $\left(197.3 \pm 11.1\right.$ vs. $182.9 \pm 11.7$ cells $/ \mathrm{mm}^{2}, t_{(7)}=0.86, p=$ $0.41)$ or the cell cycle marker Ki67 $(422.4 \pm 24.9$ vs. $371.8 \pm 30.8$ cells $\left./ \mathrm{mm}^{2}, t_{(7)}=1.27, p=0.25\right)$ were found (Fig. 1A-C), indicating no effects on cell proliferation. However, staining for the early neuronal marker doublecortin (DCX) revealed a significant increase of immunoreactive cells in the Cyfip $1^{+/-}$animals $(367.0 \pm 21.9$ vs. $550.6 \pm 39.4$ cells $/ \mathrm{mm}^{2}, t_{(15)}=4.38, p=6.21 \times 10^{-4}$, Fig. $1 \mathrm{D}, \mathrm{E})$, consistent with the presence of increased numbers of immature neurons. In a 30 day BrdU pulse-chase protocol we observed increased numbers of mature postmitotic BrdU $+/ \mathrm{NeuN}+$ labelled cells in the dentate gyrus of Cyfip $1^{+/-}$animals $\left(42.9 \pm 7.6\right.$ vs. $61.5 \pm 3.9$ cells $/ \mathrm{mm}^{2}$, $\left.t_{(10)}=4.54, p=6.8 \times 10^{-4}\right)$, as well as an increased fraction of NeuN + cells in the total BrdU+ population $\left(51.0 \pm 2.7 \% \quad\right.$ vs. $\left.\quad 60.5 \pm 0.8 \%, \quad t_{(10)}=3.95, \quad p=0.002\right)$ (Fig. $1 \mathrm{~F}-\mathrm{H}$ ). These data indicated that the excess numbers of immature neurons seen in the Cyfip $1^{+/-}$animals were maintained into maturation. We found no significant effects of sex of the animal (see Supplementary Results and Fig. S2).

\section{Increased neuronal numbers in Cyfip 1 haploinsufficiency is due to decreased apoptosis}

To investigate the cell-intrinsic effects of Cyfip1 haploinsufficiency independent of niche effects we used primary hippocampal progenitor cultures. The in vitro data corroborated the ex vivo finding, showing an increase in the fraction of DCX immunoreactive cells in the cultures prepared from Cyfip $1^{+/-}$animals $(5.5 \pm 0.5 \%$ vs. $8.8 \pm$ $1.4 \%, t_{(16)}=2.78, p=0.014$, Fig. 2A, B) in the absence of any effects on proliferation (see Supplementary Results and Fig. S3). There were no differences in the proportions of earlier progenitors of the $1 / 2 \mathrm{a}$ GFAP + /nestin + type $\left(9.4 \pm 1.2 \%\right.$ vs. $\left.10.0 \pm 1.7 \%, t_{(35)}=0.76, p=0.79\right)$, or $2 \mathrm{~b}$ GFAP-/nestin+/DCX - types $(22.1 \pm 1.6 \%$ vs. $20.2 \pm$ $2.5 \%, t_{(35)}=0.67, p=0.51$ ), (Fig. 2C-E), indicating no effects on early stages of neurogenesis. We next assessed general cell viability and survival. We saw a significant increase in the proportion of viable MitoTracker + cells $\left(82.4 \pm 1.2 \%\right.$ vs. $\left.89.6 \pm 0.7 \%, t_{(14)}=3.23, p=0.002\right)$ and fewer propidium iodine positive dead/dying cells $(7.7 \pm$ $0.6 \%$ vs. $5.4 \pm 0.5 \%, t_{(16)}=2.44, p=0.026$ ) (Fig. $2 \mathrm{~F}-\mathrm{H}$ ). This suggested an apototic mechanism. Indeed, we found significantly fewer cleaved-caspase-3 immunoreactive cells in vivo in the dentate gyrus of Cyfip $1^{+/-}$animals $\left(2.1 \pm 0.2\right.$ vs. $1.2 \pm 0.1 \mathrm{cells} / \mathrm{mm}^{2}, t_{(23)}=3.01, p=0.006$, Fig. 2I, J). To confirm this, we investigated apoptosis specifically in the DCX+ populations in our cultures. We found a marked reduction in the fraction of $\mathrm{DCX}+/$ nestin - cells positive for cleaved-caspase- $3(25.9 \pm 5.5 \%$ vs. $7.2 \pm 2.5 \%, t_{(16)}=2.32, p=0.034$, Fig. $\left.2 \mathrm{~K}, \mathrm{~L}\right)$, again demonstrating a high level of concordance between effects in cell culture and intact brain tissue. We found no sex effects in any of the phenotypes investigated in the primary cultures (see Supplementary Results and Fig. S4). Together, these data suggested Cyfip 1 haploinsufficiency impaired a normal homoeostatic apoptotic mechanism for modulating newborn neuron number, leading to a greater number surviving to go on to maturity.

\section{Evidence that apoptosis of newly born neurons is mediated by microglia secreted factors}

Given the known roles microglia play in earlier stages of neurogenesi ${ }^{53}$, we speculated that microglia may play a role in the survival and death of adult newborn immature neurons. First, we established that there were no genotype differences in density of Iba1+ microglia in hippocampus ex vivo or in vitro (Fig. S5, in either sex). To investigate the 

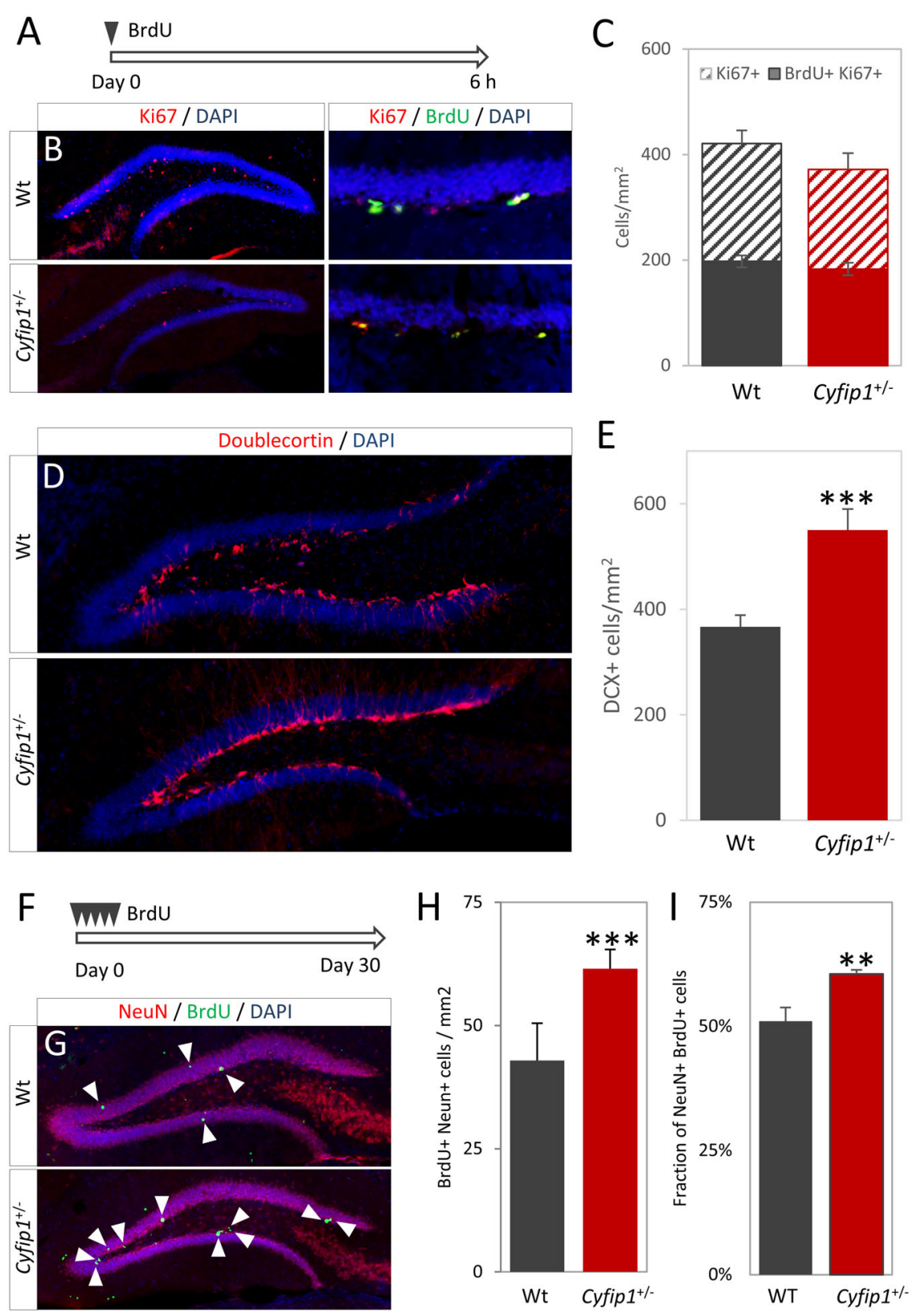

Fig. 1 Cyfip 1 haploinsufficiency increases neurogenesis in vivo in the absence of changes in proliferation. A Experimental scheme, proliferation rates were studied with a $6 \mathrm{~h}$ pulse of BrdU. B Representative immunohistochemistry showing distribution and location of proliferative $(\mathrm{Ki} 67+)$ and dividing (Ki67+BrdU+) cells in wild-type and Cyfip ${ }^{+/-}$animals $(n=4$ each), respectively. C Quantification showed no difference in numbers of proliferative cells or number of cell divisions. D Representative staining for DCX in wild-type and Cyfip $1^{+/-}$animals $(n=8$ each), respectively. E Quantification showed a significantly larger number of immature neurons in the Cyfip $1^{+/-}$animals. $\mathbf{F}$ Experimental scheme, cell maturation was studied with a 30 day BrdU pulse-chase. G Representative images showing BrdU+ and NeuN+ cells. Arrowheads indicate BrdU+

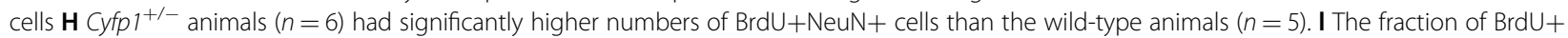
cells which had matured into NeuN+ cells was significantly increased in the Cyfip $1^{+/-}$animals. All data are shown as mean \pm SEM.

role of microglia in neuronal apoptosis, we depleted microglia from wild-type primary hippocampal progenitor cultures using the specific microglia toxin Mac-1-SAP, targeting CD11b expressing cells. We established the efficacy of the toxin against primary mouse microglia (Fig. 3A).
Depletion of microglia from hippocampal cultures led to an increase in the proportion of DCX + cells $(7.5 \pm 1.6 \%$ vs. $12.7 \pm 0.3 \%, t_{(7)}=3.19, p=0.019$, Fig. $\left.3 \mathrm{~B}\right)$, and a specific decrease in the fraction of apoptotic DCX + cells $\left(13.1 \pm 3.1 \%\right.$ vs. $2.7 \pm 1.7 \%, t_{(7)}=2.93, p=0.026$, Fig. $\left.3 \mathrm{C}\right)$, 


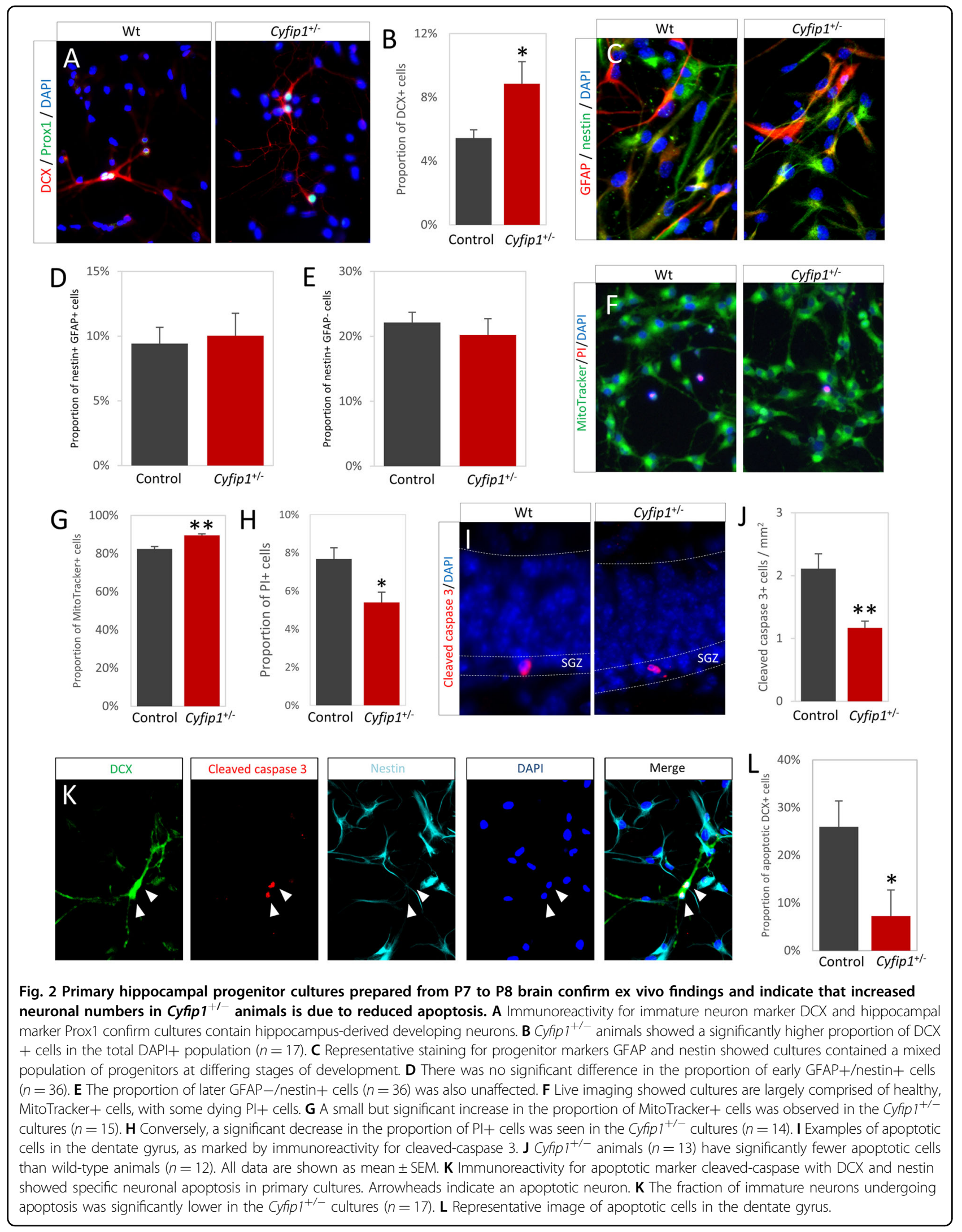




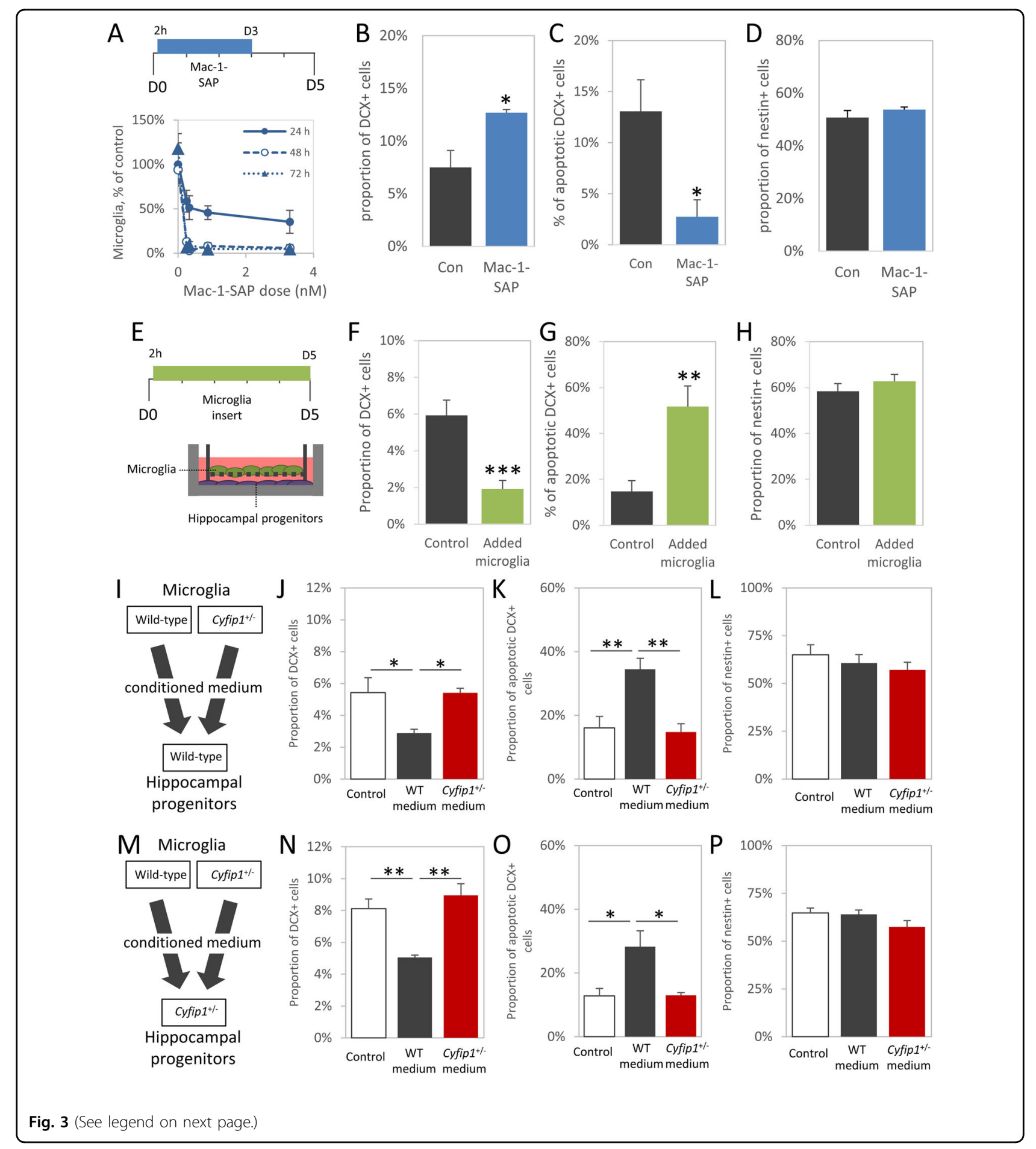

without any associated changes in the nestin + population $\left(50.7 \pm 2.7 \%\right.$ vs. $53.8 \pm 1.0 \%, t_{(7)}=1.07, p=0.32$, Fig. $\left.3 \mathrm{D}\right)$, phenocopying the Cyfip $1^{+/-}$cultures, and showing a significant role for microglia in regulating DCX numbers. Conversely, addition of exogenous microglia on a semipermeable membrane, allowing only for soluble factors to influence neuronal apoptosis, had opposite effects. Under these conditions we observed, a decreased proportion of DCX + cells $\left(5.9 \pm 0.8 \%\right.$ vs. $1.9 \pm 0.5 \%, t_{(15)}=4.36, p=$ $6.5 \times 10^{-4}$, Fig. 3F), increased apoptosis in the DCX+ population $\left(14.8 \pm 4.7 \%\right.$ vs. $51.7 \pm 9.0 \%, t_{(15)}=3.27, p=$ 0.006 , Fig. 3G) and no effect on the nestin + population 
(see figure on previous page)

Fig. 3 Cyfip 1 haploinsufficiency affects the ability if microglia to regulate number and apoptosis rates of immature neurons. A Experimental setup, Mac-1-Sap was present in wild-type hippocampal progenitor cultures from $2 \mathrm{~h}$ after isolation until 3 days in vitro. Dose-response curves of microglial death after Mac-1-SAP administration show near-complete depletion of Iba1+ microglia was achieved within $48 \mathrm{~h}(n=6)$. B Cultures where microglia were depleted $(n=4)$ showed a higher proportion of DCX+cells than controls $(n=4)$. C Correspondingly, the fraction of DCX cells undergoing apoptosis, as marked by cleaved-caspase 3 staining, was significantly decreased in the absence of microglia. D The proportion of nestin+ cells was unaffected by microglia depletion. E Experimental setup, membrane inserts containing wild-type microglia prepared from P7-8 brain were added to cultures $2 \mathrm{~h}$ after progenitor isolation and were present throughout the remainder of the experiment. Due to the experimental conditions, no cell-cell contact between microglia and progenitors was possible, but secreted factors were free to diffuse. $\mathbf{F}$ The presence of microgliacontaining inserts $(n=8)$ significantly decreased the proportion of DCX expressing cells compared to controls $(n=8)$. $\mathbf{G}$ Conversely, the added microglia significantly increased apoptosis in DCX+ cells. $\mathbf{H}$ No effect was seen in nestin expressing cells. I Experimental design, wild-type hippocampal progenitors were exposed to conditioned medium from either wild-type or Cyfip $1^{+/-}$microglia. $\mathbf{J}$ In wild-type cultures, conditioned medium from wild-type microglia induced a significant reduction in the proportion of DCX + cells, whereas medium from Cyfip $1^{+/-}$microglia was unable to do this ( $n=4$ in all conditions). $\mathbf{K}$ An inverse pattern was seen in neuronal apoptosis where wild-type microglia medium increased apoptosis in DCX+ immature neurons but Cyfip $1^{+/-}$medium did not. L Nestin+ cells were not significantly affected by the presence of microglial

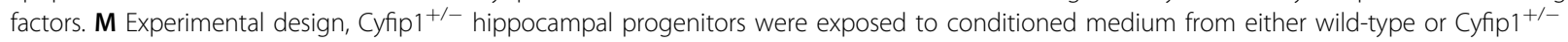
microglia. $\mathbf{N}$ As seen in the wild-type cultures, a significant decrease in DCX+ cells was observed only after stimulation with medium from wild-type microglia ( $n=4$ in all conditions). $\mathbf{O}$ Similarly, medium from Cyfip $1^{+/-}$microglia was unable to induce apoptosis in Cyfip $1^{+/-}$progenitors. P Again, the nestin+ population was unaffected. All data are shown as mean \pm SEM.

$\left(58.4 \pm 3.3 \%\right.$ vs. $62.7 \pm 3.0 \%, t_{(15)}=0.98, p=0.34$, Fig. $\left.3 \mathrm{H}\right)$. These experiments provide converging evidence for a previously unknown function of microglia in inducing apoptosis of immature neurons in the hippocampus, which is mediated, at least in part, by soluble factors.

\section{Haploinsufficiency of Cyfip 1 inhibits the ability of microglia to support apoptosis of newborn immature hippocampal neurons}

Having established a novel microglial dependent mechanism for regulation of neuronal survival, we next tested whether effects on this process could underlie the effects of Cyfip1 haploinsufficiency on neuronal survival. To directly address the potential role the induction of apoptosis by microglial secreted factors in an unbiased way, we used a cross-genotype conditioned medium approach. Medium obtained from isolated wild-type and Cyfip $1^{+/-}$microglia preparations was added to both the wild-type and Cyfip1 $1^{+/-}$hippocampal progenitor enriched cultures (see Fig. 3I, M for schematic of design) and cell proportions and levels of apoptosis were determined. In the wild-type progenitor cultures, adding conditioned medium from wild-type microglia resulted in a significant reduction in the proportion of DCX + cells, compared to unstimulated cells, whereas Cyfip $1^{+/-}$conditioned medium had no effect ( $5.4 \pm 0.9 \%$ vs. $2.9 \pm 0.2 \%$ vs. $5.4 \pm 0.3 \%$ for unstimulated, wild-type

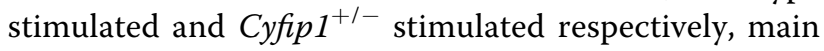
effects of genotype $F_{(2,9)}=6.31, p=0.019$, Fig. 3J). Post hoc testing confirmed significant differences between controls and wild-type medium $(p=0.031)$ and between those stimulated with wild-type and Cyfip $1^{+/-}$medium $(p=0.033)$. This pattern of effects was mirrored inversely in the fraction of DCX + cells that were also immunopositive for cleaved-caspase-3, with only conditioned medium from wild-type microglia cultures increasing apoptosis $(16.1 \pm 3.6 \%$ vs. $34.5 \pm 3.5 \%$ vs. $14.8 \pm 2.5 \%$, main effects of genotype $F_{(2,9)}=11.57, p=$ 0.003 , Fig. $3 \mathrm{~K})$. Again, post hoc testing showed the wildtype stimulated condition was significantly different from controls and Cyfip $1^{+/-}$stimulated condition $(p=$ 0.007 and $p=0.005$, respectively). Consistent with the conclusion that these effects were limited to the DCX+ immature neuron population of cells, the proportion of cultures that was nestin + was unchanged across all conditions $(65.0 \pm 5.2 \%$ vs. $60.7 \pm 4.4 \%$ vs. $57.1 \pm 4.1 \%$, $F_{(2,9)}=0.75, p=0.50$, Fig. 3L).

Importantly, identical patterns of effects were seen in the Cyfip1 $1^{+-}$hippocampal progenitor cultures. Using an identical experimental design (Fig. 3M), we found that conditioned medium from wild-type microglia, but not from Cyfip $1^{+/-}$microglia, decreased the proportion of DCX + cells $(8.1 \pm 0.6 \%$ vs. $5.1 \pm 0.2 \%$ vs. $9.0 \pm 0.7 \%$, main effects of genotype $F_{(2,9)}=13.82, p=0.002$, Fig. $3 \mathrm{~N}$ ) with post hoc analysis confirming wild-type microglia medium stimulated conditions were significantly different from control and Cyfip1 $1^{+/-}$stimulated conditions $(p=0.008$ and $p=0.002$, respectively). Similarly, only conditioned medium from wild-type microglia was associated with a significant increase in the proportion of apoptotic DCX+ cells $(12.8 \pm 2.3 \%$ vs. $28.3 \pm 5.0 \%$ vs. $13.0 \pm 0.9 \%$, main effects of genotype $F_{(2,9)}=6.12, p=0.021$, Fig. 3O) with post hoc analysis confirming the wild-type stimulated condition was significantly different from the unconditioned medium controls and Cyfip $1^{+/-}$stimulated condition ( $p=0.007$ and $p=0.005$, respectively). As before, no effect of any of the medium conditions was seen on the nestin-positive population $(64.7 \pm 2.6 \%$ vs. $64.0 \pm 2.3 \%$ vs. $57.5 \pm 3.3 \%, F_{(2,9)}=2.13, p=0.18$, Fig. 3P). These data provide converging evidence for induction of neuronal 
apoptosis through factors secreted by microglia a process which is disrupted in Cyfip1 $1^{+/-}$animals.

\section{Proliferative cells are abnormally positioned in the Cyfip ${ }^{+/-}$hippocampus}

As well as having to survive, newborn cells have to migrate to the correct location to functionally integrate into existing hippocampal circuitry. We noted that the gross positioning of newborn cells appeared different in the Cyfip1 $1^{+/-}$animals. Cells undergoing proliferation are normally largely limited to the subgranular zone (SGZ) of the dentate gyrus, apart from some radially migrating neuroblasts undergoing their final divisions. Indeed, the majority of Ki67 + cells were found in the SGZ, however a significantly larger fraction was found in the SGZ in the Cyfip $1^{+/-}$animals (Fig. 4A, B, $80.4 \pm 1.1 \%$ vs. $88.2 \pm 0.6 \%$, $\left.t_{(14)}=6.001, p=3.2 \times 10^{-5}\right)$. As well as an increased fraction remaining in the SGZ, Cyfip $1^{+/-} \mathrm{Ki67}+$ cells were also found significantly less far out into the granular zone (Fig. $4 \mathrm{C}, 17.0 \pm 1.4 \mu \mathrm{m}$ vs. $11.9 \pm 1.0 \mu \mathrm{m}, t_{(14)}=$ $2.999, p=0.009)$. These data suggested a migration deficit in Cyfip $1^{+/-}$animals.

\section{Cyfip $1^{+/-}$adult-born neurons show impaired migration into the granular layer}

In order to gain direct evidence of abnormal migration in Cyfip $1^{+/-}$animals we tracked the final positioning of newly born neurons using a 30 day BrdU pulse-chase paradigm followed by staining for BrdU/Neun. Neurons born in the SGZ normally undergo a radial migration outwards into the granular layer. Consistent with a migration deficit in the Cyfip1 $1^{+-}$animals we found a greater proportion of $\mathrm{BrdU}+\mathrm{NeuN}+$ cells had remained in the SGZ in (Fig. 4D, E, $52.2 \pm 3.6 \%$ vs. $66.7 \pm 2.8 \%$, $\left.t_{(12)}=3.197, p=0.008\right)$ and those cells that had migrated had moved a smaller distance into the granular zone (Fig. $4 \mathrm{~F}, 13.6 \pm 2.3 \mu \mathrm{m}$ vs. $\left.6.0 \pm 0.7 \mu \mathrm{m}, t_{(12)}=3.549, p=0.004\right)$. When we further analysed the location of the BrdU+ $/ \mathrm{NeuN}+$ cells that had left the SGZ by dividing the dentate gyrus into four quartiles mapping on to the route taken by migrating cells, we found that the distribution of cells over these quartiles was significantly different (Fig. S6, $x^{2}=25.7, p=1.1 \times 10^{-5}$ ) and, as would be anticipated with a migration deficit, BrdU $+/ \mathrm{NeuN}+$ cells in the Cyfip $1^{+/-}$animals were skewed towards the two most proximal quartiles (i.e. those closest to the starting point in the SGZ). Finally, we examined the putative migration phenotype observed in vivo by performing time-lapse imaging in primary hippocampal cultures. Over the $2 \mathrm{~h}$ imaging period, Cyfip $1^{+/-}$cells covered significantly less distance than wild-type cells (Fig. 4G, H, $133.0 \pm 4.7$ vs. $\left.90.7 \pm 12.0 \mu \mathrm{m}, t_{(10)}=3.993, p=0.003\right)$. Both the in vivo and in vitro findings were consistent with a migration deficit due to Cyfip1 haploinsufficiency.
Actin dynamics are altered in Cyfip $1^{+/-}$cells and inhibition of Arp2/3 activity rescues migration deficits in vitro

As CYFIP1 contributes to the regulation of actin branching and polymerisation through the WAVE1 complex, a function crucial for cytoskeletal reorganisation and cell migration, we speculated that the Cyfip1 $1^{+/-}$ related migration phenotype was related to alterations in actin dynamics. A main determinant of actin function in the context of cytoskeleton reorganisation is the ratio of filamentous (F) to globular (G) actin. We first checked this ratio in primary hippocampal cultures, staining with fluorescently labelled phalloidin for F-actin and DNAse1 for G-actin, and found a significant increase in F-G-actin ratio in Cyfip $1^{+/-}$cells (Fig. 5A, B, $0.67 \pm 0.3$ vs. $1.48 \pm$ $\left.0.28, t_{(5)}=2.904, p=0.034\right)$. An increase in F-G-actin ratio would be consistent with concurrent increased activity in Arp2/3 brought about by an expected reduction of CYFIP1-WAVE1 complexing under conditions of Cyfip1 haploinsufficiency. Hence, we further speculated that treatment with Arp2/3 inhibitors would influence, and potentially rescue, the abnormal cell migration phenotypes in Cyfip $1^{+/-}$cultures. To do this we used two drugs; CK-548, which induces a conformation change inhibiting actin binding and CK-666, which stabilises the inactive form of Arp2/3.

Analysis by time-lapse imaging showed that both drugs were effective in influencing cell migration. However, the effects were essentially opposite in wild-type and Cyfip $1^{+/-}$cultures. In wild-type cultures, treatment with Arp2/3 inhibitors had a significant effect on migration (Fig. 5C, vehicle: $107.3 \pm 5.6$, CK-548:76.3 \pm 3.9 , CK-666: $\left.65.2 \pm 5.4 \mu \mathrm{m}, F_{(2,24)}=18.91, p=1.17 \times 10^{-5}\right)$, with both CK-548 and CK-666 showing significant decreases in distance moved $\left(p=5.8 \times 10^{-4}\right.$ and $1.1 \times 10^{-5}$, respectively). In $C y f i p 1^{+/-}$cultures, there was also a significant effect on migration by Arp $2 / 3$ inhibitors (Vehicle: 68.1.3 \pm 4.4, CK-548:117.2 \pm 8.8 , CK-666:112.0 $\pm 9.8 \mu \mathrm{m}$, $\left.F_{(2,6)}=11.39, p=0.009\right)$. In contrast to wild-type cultures, both CK-548 and CK-666 caused significant increases in distance moved $(p=0.011$ and $p=0.019$, respectively), restoring migration to wild-type levels and suggesting excessive Arp2/3 activity was responsible for the migration deficit in Cyfip $1^{+/-}$cells.

\section{Discussion}

We have shown changes in the survival and migration of newly born neurons in hippocampus in a model of Cyfip1 haploinsufficiency, recapitulating the lowered gene dosage found in the pathogenic 15q11.2(BP1-BP2) copy number deletion. We report that Cyfip $1^{+/-}$animals have larger numbers of surviving newly born neurons in hippocampus, in the absence of increases in numbers of stem cells or rate of proliferation, and provide evidence that this is the result of a specific failure in microglia-induced 

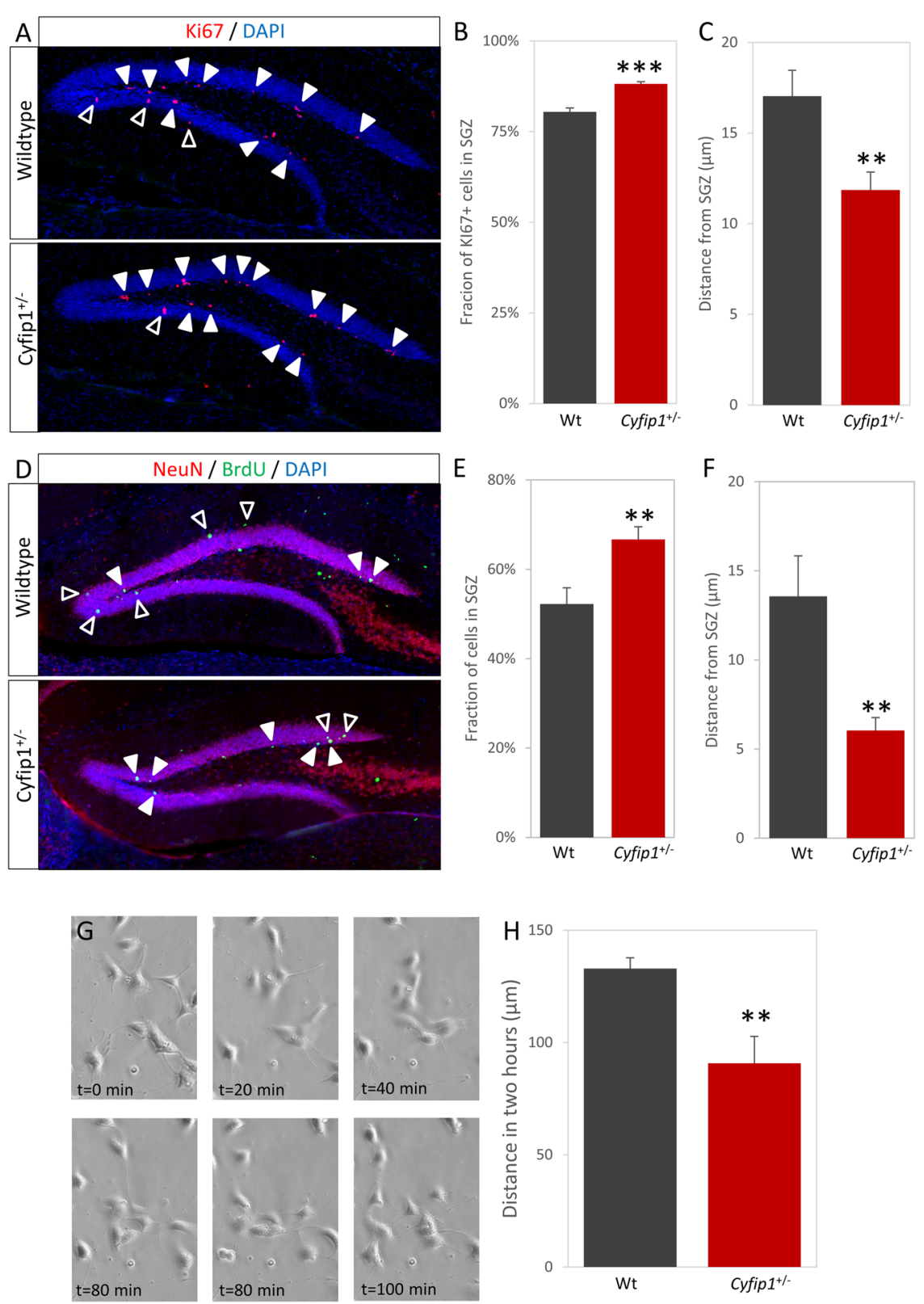

Fig. 4 Altered migration of immature Cyfip $1^{+/-}$neurons. A Immunohistochemistry for the Ki67, showing cells in the cell cycle, shows the majority are located in the SGZ (closed arrowheads), but some are starting to move out into the granular layer (open arrowheads). B A significantly larger fraction of Ki67+ cells are located in the SGZ in Cyfip $1^{+/-}$animals, comparted to wild types. C The distance moved from the SGZ is significantly lower in the Cyfip $1^{+/-}$animals, comparted to wild types. D Post-mitotic adult-born neurons (NeuN+/BrdU+) are located predominantly in the granular layer (open arrowheads), but some are still located in or near the SGZ (closed arrowheads). E As with Ki67+ cells, a smaller fraction of Cyfip ${ }^{+/-}$cells are in the granular layer. $\mathbf{F}$ Distance moved by these cells is significantly lower. $\mathbf{G}$ Time-lapse imagining shows the movement of cells in primary hippocampal cultures. $\mathbf{H}$ Quantification of cell movement during live imaging shows a significantly smaller distance moved by the Cyfip $1^{+/-}$cells.

apoptosis (see summary of proposed mechanism in Fig. S7). These newly born neurons also show an Arp2/3 dependent deficit in migration, resulting in a failure to reach their appropriate locations within the dentate gyrus.

Our study is the first to our knowledge to demonstrate a direct effect of microglial soluble factors on the survival of immature neurons as a homoeostatic mechanism. In contrast to earlier findings examining the effect of microglia on adult neurogenesis ${ }^{54-56}$, we show this occurs in the absence of overt inflammatory disease or microglial activation. Our data indicate this is a mechanism susceptible to pathogenic changes, as evidenced by the effects of Cyfip1 haploinsufficiency on the ability of microglia to regulate apoptosis of immature neurons. 

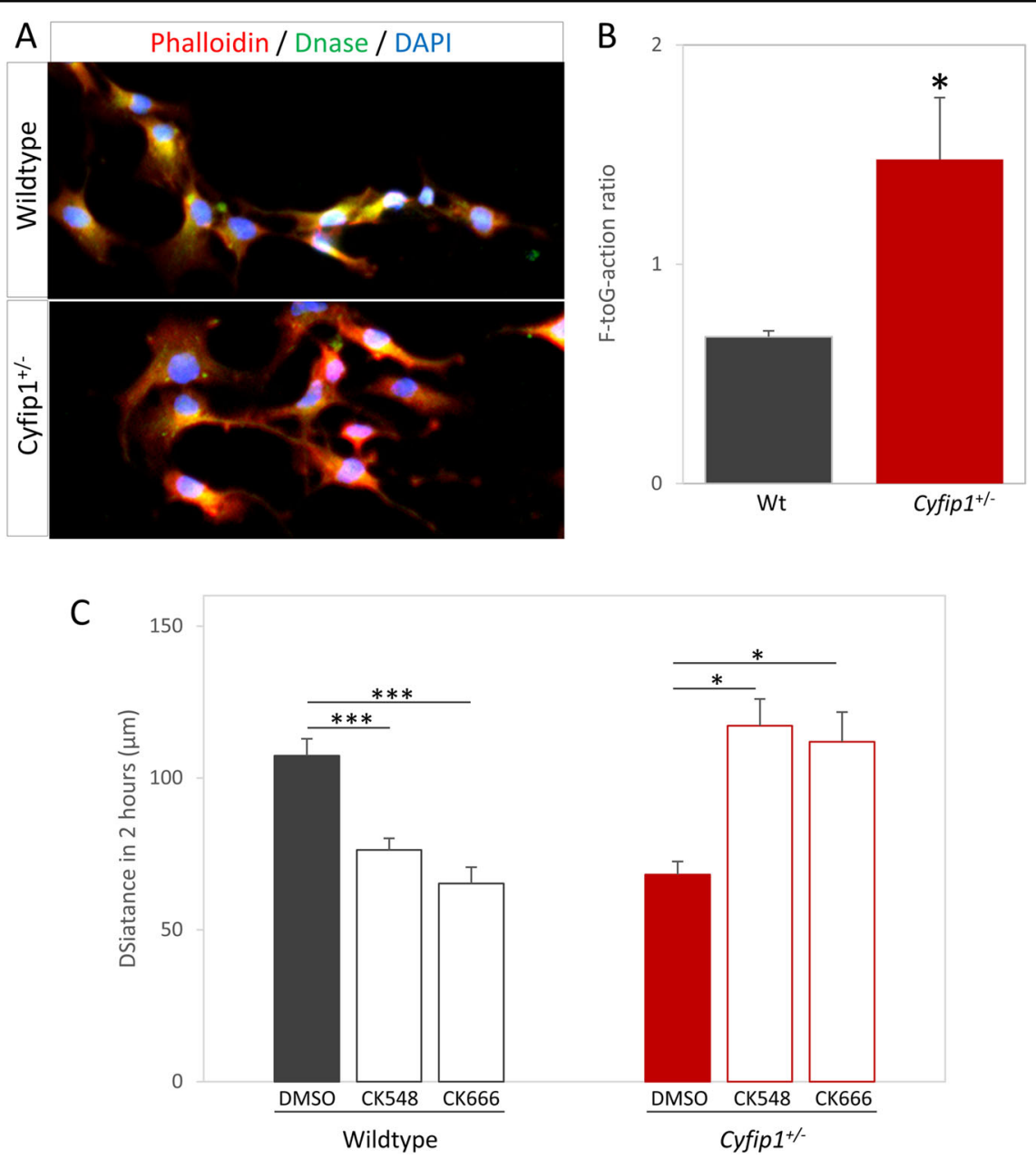

Fig. 5 Disruption of Arp2/3 mediated actin dynamics is responsible for migration deficit. A Fluorescently labelled phalloidin and DNAse show the amount of filamentous and globular actin, respectively. B Quantification of fluorescence intensity shows Cyfip $1^{+/-}$cells have an increased F- to Gactin ratio. C Treatment with Arp2/3 inhibitors CK-548 or CK-666 in wild-type cells significantly reduces the distance moved by cells during live imagine, whereas treatment of Cyfip $1^{+/-}$cells restores distances to wild-type levels.

The effects of Cyfip1 haploinsufficiency were highly cell-type specific and appeared limited to the stage of cell maturity where neuronal progenitors are fully committed to a neuronal phenotype, a stage of development characterised by high levels of apoptosis. Whilst we have demonstrated that soluble factors from microglia are involved in controlling numbers of immature neurons during adult neurogenesis, and that haploinsufficiency of Cyfip1 leads to reduced apoptosis via an effect on factors secreted from microglia, the soluble factor(s) responsible remain to be identified. Future proteomic and pharmacological work will serve to identify and validate the identity of the responsible medatior(s). Cyfip1 has pleotropic roles but the potential links between CYFIP1, actin remodelling. and cytokine secretion are especially interesting $^{57}$. A focus for CYFIP1 action in microglia gains further evidence from a recent preprint by showing the effect of cell specific deletion of Cyfip1 on microglia morphology, movement and function ${ }^{58}$.

In parallel, we have shown that adult-born neurons in Cyfip $1^{+/-}$animals show a significantly impaired migration phenotype. Here our data point to a key role for CYFIP1 effects on actin dynamics mediated by Arp2/3 activity. We hypothesise that lowered dosage will lead to reduced engagement of CYFIP protein with the CYFIPWAVE1-Arp2/3 pathway resulting in reduced suppression of Arp $2 / 3$ activity and abnormal actin cytoskeleton remodelling required for migration. We show evidence to support this hypothesis though, as predicted, an increase in F-G-actin ratio and rescue of the migration phenotype by pharmacological inhibition of Arp $2 / 3$ activity. The activation of the WAVE-Arp $2 / 3$ system is complex and, can take place in at least two different Rac1 mediated pathways $^{59,60}$. It is unlikely that CYFIP1 is the sole 
regulator of this activation, but our data point towards a significant role in this process.

The combined effects of failure to undergo homoeostatic apoptosis and failure to migrate to the correct location is likely to have profound effects on the functionality of adult-born neurons in hippocampus.. We observed lasting effects up to 30 days post division, when adult-born neurons will already have local connections $^{61}$. The prolonged survival and expression of mature markers of these ectopic cells makes it likely these cells will be active, indeed, it is known that synaptic NMADR signalling is required for neuronal survival prior to this developmental timepoint ${ }^{62}$, making it likely these cells are integrated in some form in the local circuitry. The exact functional consequences on a network level are difficult to predict and will require further electrophysiological study, both on the individual cell and network level. Haploinsufficiency of Cyfip1 has been shown to have disease relevant behavioural consequences in both mice ${ }^{63,64}$ and rat $^{65}$ models, but to date, much of the neurobiology and behaviour in these models has remained unexplored. Our data would indicate a focus on hippocampal circuitry would prove of interest. Neuronal migration in the hippocampus has, to our knowledge, not been examined in patient populations. However, as AHN does take place in humans, and the biochemical pathways relevant to this study are well conserved, it is likely similar deficits would be observed in $15 \mathrm{q} 11.2 \mathrm{BP} 1-\mathrm{BP} 2$ carriers.

The 15q11.2(BP1-BP2) deletion is not solely associated with schizophrenia, it also markedly increases risk for autism spectrum disorders ${ }^{66}$, ADHD and developmental delay $^{67}$ and epilepsy ${ }^{68}$. Whether altered microglia functioning due to Cyfip1 haploinsufficiency, impacting on AHN, also plays a part in these disorders is an open question, but all have been associated with alterations in immune system and microglia functioning ${ }^{69,70}$. Likewise, alterations in migration would have equally profound consequences in other disorders.

As noted previously, a number of other schizophrenia risk genes have also been shown to modify AHN in model systems. Whilst the effects are distinct from those of Cyfip1, each risk gene impacting on neurogenesis in its own way, this convergence of function may be indicative of a common pathogenic route for a number of diverse psychiatric risk genes.

\footnotetext{
Acknowledgements

The authors would like to thank Chen Liang and Manal Adam for assistance with dissections, Caroline Best for assistance with genotyping and Joe Davids for initial pilot immunohistochemistry. This work was supported by Wellcome Trust Strategic Award 100202/Z/12/Z (DEFINE) and the Hodge Foundation, UK. L.J.W. was supported by a Waterloo Foundation Early Career Research Fellowship.
}

\begin{abstract}
Author details
${ }^{1}$ Neuroscience and Mental Health Research Institute, Cardiff University, Hadyn Ellis Building, Maindy Road, Cardiff, UK. ${ }^{2}$ Brain Repair and Intercranial Neurotherapeutics Unit, Cardiff University, Hadyn Ellis Building, Maindy Road, Cardiff, UK. ${ }^{3}$ Hodge Centre for Neuropsychiatric Immunology, Cardiff University, Hadyn Ellis Building, Maindy Road, Cardiff, UK. ${ }^{4}$ School of Psychology, Cardiff University, Tower Building, Cardiff, UK
\end{abstract}

\section{Author contributions}

N.H. and L.S.W. designed the study. N.H. designed and performed experiments, and drafted the paper. J.C. performed animal husbandry/genotyping. L.J.W. optimised and assisted with primary culture. W.P.G. and L.S.W. initiated the study and assisted with data interpretation. M.J.O., W.P.G., J.H. and L.S.W. critically appraised the manuscript. All authors approved the final paper.

\section{Conflict of interest}

The authors declare no competing interests.

\section{Publisher's note}

Springer Nature remains neutral with regard to jurisdictional claims in published maps and institutional affiliations.

Supplementary information The online version contains supplementary material available at https://doi.org/10.1038/s41398-021-01415-6.

Received: 26 August 2020 Revised: 21 April 2021 Accepted: 4 May 2021 Published online: 24 May 2021

\section{References}

1. Pardiñas, A. F. et al. Common schizophrenia alleles are enriched in mutationintolerant genes and in regions under strong background selection. Nat. Genet. https://doi.org/10.1038/s41588-018-0059-2 (2018).

2. Rees, E. et al. Analysis of copy number variations at 15 schizophreniaassociated loci. Br. J. Psychiatry 204, 108-114 (2014).

3. Hall, J. et al. Genetic risk for schizophrenia: convergence on synaptic pathways involved in plasticity. Biol. Psychiatry 77, 52-58 (2015).

4. Network and Pathway Analysis Subgroup of Psychiatric Genomics Consortium. Psychiatric genome-wide association study analyses implicate neuronal, immune and histone pathways. Nat. Neurosci. 18, 199-209 (2015).

5. Cox, D. M. \& Butler, M. G. The 15q11.2 BP1-BP2 microdeletion syndrome: a review. Int J. Mol. Sci. 16, 4068-4082 (2015).

6. Stefansson, $\mathrm{H}$. et al. Large recurrent microdeletions associated with schizophrenia. Nature 455, 232-236 (2008).

7. Kirov, G. et al. Support for the involvement of large copy number variants in the pathogenesis of schizophrenia. Hum. Mol. Genet. 18, 1497-1503 (2009).

8. Zhao, Q. et al. Rare CNVs and tag SNPs at $15 q 11.2$ are associated with schizophrenia in the Han Chinese population. Schizophr. Bull. 39, 712-719 (2013).

9. Pathania, M. et al. The autism and schizophrenia associated gene CYFIP1 is critical for the maintenance of dendritic complexity and the stabilization of mature spines. Transl. Psychiatry 4, e374 (2014).

10. Oguro-Ando, A. et al. Increased CYFIP1 dosage alters cellular and dendritic morphology and dysregulates mTOR. Mol. Psychiatry 20, 1069-1078 (2015).

11. Hsiao, K. et al. Cyfip1 regulates presynaptic activity during development. J. Neurosci. 36, 1564-1576 (2016).

12. Darnell, J. C. et al. FMRP stalls ribosomal translocation on mRNAs linked to synaptic function and autism. Cell 146, 247-261 (2011).

13. Chen, Z. et al. Structure and control of the actin regulatory WAVE complex. Nature 468, 533-538 (2010).

14. Spalding, K. L. et al. Dynamics of hippocampal neurogenesis in adult humans Cell 153, 1219-1227 (2013).

15. Kempermann, G., Song, H. \& Gage, F. H. Neurogenesis in the adult hippocampus. Cold Spring Harb. Perspect. Biol. 7, a018812 (2015).

16. Clelland, C. D. et al. A functional role for adult hippocampal neurogenesis in spatial pattern separation. Science 325, 210-213 (2009).

17. Sahay, A. et al. Increasing adult hippocampal neurogenesis is sufficient to improve pattern separation. Nature 472, 466-470 (2011). 
18. Kee, N., Teixeira, C. M., Wang, A. H. \& Frankland, P. W. Preferential incorporation of adult-generated granule cells into spatial memory networks in the dentate gyrus. Nat. Neurosci. 10, 355-362 (2007).

19. Dupret, D. et al. Spatial relational memory requires hippocampal adult neurogenesis. PLOS ONE 3, e1959 (2008).

20. Akers, K. G. et al. Hippocampal neurogenesis regulates forgetting during adulthood and infancy. Science 344, 598-602 (2014).

21. Seo, D.-O. et al. Adult hippocampal neurogenesis modulates fear learning through associative and nonassociative mechanisms. J. Neurosci. 35, 11330-11345 (2015).

22. Deng, W., Saxe, M. D., Gallina, I. S. \& Gage, F. H. Adult-born hippocampal dentate granule cells undergoing maturation modulate learning and memory in the brain. J. Neurosci. 29, 13532-13542 (2009).

23. Martinelli, C. \& Shergill, S. S. Clarifying the role of pattern separation in schizophrenia: the role of recognition and visual discrimination deficits. Schizophr. Res. 166, 328-333 (2015).

24. Das, T. et al. Loss of pattern separation performance in schizophrenia suggests dentate gyrus dysfunction. Schizophr. Res. 159, 193-197 (2014).

25. Salgado-Pineda, P. et al. Examining hippocampal function in schizophrenia using a virtual reality spatial navigation task. Schizophr. Res. 172, 86-93 (2016).

26. Holt, D. J. et al. Extinction Memory Is Impaired in Schizophrenia. Biol. Psychiatry 65, 455-463 (2009).

27. Newton, S. S. \& Duman, R. S. Neurogenic actions of atypical antipsychotic drugs and therapeutic implications. CNS Drugs 21, 715-725 (2007).

28. Ye, F. et al. DISC1 regulates neurogenesis via modulating kinetochore attachment of Ndel1/Nde1 during mitosis. Neuron 96, 1041-1054.e5 (2017).

29. Chen, Q. et al. SREB2/GPR85, a schizophrenia risk factor, negatively regulates hippocampal adult neurogenesis and neurogenesis-dependent learning and memory. Eur. J. Neurosci. 36, 2597-2608 (2012).

30. Temme, S. J., Bell, R. Z., Fisher, G. L. \& Murphy, G. G. Deletion of the mouse homolog of CACNA1C disrupts discrete forms of hippocampal-dependent memory and neurogenesis within the dentate gyrus. eNeuro $\mathbf{3}$ https://doi.org/ 10.1523/ENEURO.0118-16.2016 (2016)

31. Moon, A. L. et al. CACNA1C: Association With Psychiatric Disorders, Behavior, and Neurogenesis. Schizophr. Bull. https://doi.org/10.1093/schbul/sby096 (2018).

32. Ouchi, Y. et al. Reduced adult hippocampal neurogenesis and working memory deficits in the Dgcr8-deficient mouse model of 22q11.2 deletionassociated schizophrenia can be rescued by IGF2. J. Neurosci. 33, 9408-9419 (2013).

33. Smrt, R. D. et al. MicroRNA miR-137 regulates neuronal maturation by targeting ubiquitin ligase mind bomb-1. Stem Cells 28, 1060-1070 (2010).

34. Barbeau, D. et al. Decreased expression of the embryonic form of the neural cell adhesion molecule in schizophrenic brains. Proc. Natl Acad. Sci. U.S.A. 92, 2785-2789 (1995).

35. Reif, A. et al. Neural stem cell proliferation is decreased in schizophrenia, but not in depression. Mol. Psychiatry 11, 514-522 (2006).

36. Allen, K. M., Fung, S. J. \& Shannon Weickert, C. Cell proliferation is reduced in the hippocampus in schizophrenia. Aust. N. Z. J. Psychiatry 50, 473-480 (2016).

37. Brown, A. S. \& Derkits, E. J. Prenatal infection and schizophrenia: a review of epidemiologic and translational studies. Am. J. Psychiatry 167, 261-280 (2010).

38. Knuesel, I. et al. Maternal immune activation and abnormal brain development across CNS disorders. Nat. Rev. Neurol. 10, 643-660 (2014).

39. Miller, B. J. et al. Meta-analysis of cytokine alterations in schizophrenia: clinical status and antipsychotic effects. Biol. Psychiatry 70, 663-671 (2011).

40. Trépanier, M. O. et al. Postmortem evidence of cerebral inflammation in schizophrenia: a systematic review. Mol. Psychiatry 21, 1009-1026 (2016).

41. Bloomfield, P. S. et al. Microglial activity in people at ultra high risk of psychosis and in schizophrenia: an [ ${ }^{11}$ C]PBR28 PET brain imaging study. Am. J. Psychiatry 173, 44-52 (2016).

42. Holmes, S. E. et al. In vivo imaging of brain microglial activity in antipsychoticfree and medicated schizophrenia: a [11C](R)-PK11195 positron emission tomography study. Mol. Psychiatry 21, 1672-1679 (2016).

43. Notter, T. et al. Translational evaluation of translocator protein as a marker of neuroinflammation in schizophrenia. Mol. Psychiatry 23, 323-334 (2018).

44. Notter, T., Coughlin, J. M., Sawa, A. \& Meyer, U. Reconceptualization of translocator protein as a biomarker of neuroinflammation in psychiatry. Mol. Psychiatry 23, 36-47 (2018).
45. Paolicelli, R. C. et al. Synaptic pruning by microglia is necessary for normal brain development. Science 333, 1456-1458 (2011).

46. Schafer, D. P. et al. Microglia sculpt postnatal neural circuits in an activity and complement-dependent manner. Neuron 74, 691-705 (2012).

47. Kim, H.-J. et al. Deficient autophagy in microglia impairs synaptic pruning and causes social behavioral defects. Mol. Psychiatry 22, 1576-1584 (2017).

48. Li, Y. et al. Reciprocal regulation between resting microglial dynamics and neuronal activity in vivo. Dev. Cell 23, 1189-1202 (2012).

49. Cunningham, C. L., Martinez-Cerdeno, V. \& Noctor, S. C. Microglia regulate the number of neural precursor cells in the developing cerebral cortex. J. Neurosci. 33, 4216-4233 (2013)

50. Brown, G. C. \& Neher, J. J. Microglial phagocytosis of live neurons. Nat. Rev. Neurosci. 15, 209-216 (2014).

51. Haan, N. et al. Fgf10-expressing tanycytes add new neurons to the appetite/ energy-balance regulating centers of the postnatal and adult hypothalamus. J. Neurosci. 33, 6170-6180 (2013).

52. Meijering, E., Dzyubachyk, O. \& Smal, I. Methods for cell and particle tracking. In: Methods in Enzymology. Academic Press Inc., 2012, pp 183-200.

53. Ziv, Y. et al. Immune cells contribute to the maintenance of neurogenesis and spatial learning abilities in adulthood. Nat. Neurosci. 9, 268-275 (2006).

54. Kohman, R. A. et al. Effects of minocycline on spatial learning, hippocampal neurogenesis and microglia in aged and adult mice. Behav. Brain Res. 242, 17-24 (2013)

55. Mattei, D. et al. Minocycline rescues decrease in neurogenesis, increase in microglia cytokines and deficits in sensorimotor gating in an animal model of schizophrenia. Brain Behav. Immun. 38, 175-184 (2014).

56. Biscaro, B. et al. Inhibition of microglial activation protects hippocampal neurogenesis and improves cognitive deficits in a transgenic mouse model for Alzheimer's disease. Neurodegener. Dis. 9, 187-198 (2012).

57. Chemin, K. et al. Cytokine secretion by CD4+ T cells at the immunological synapse requires Cdc42-dependent local actin remodeling but not microtubule organizing center polarity. J. Immunol. 189, 2159-2168 (2012).

58. Drew, J. et al. Control of microglial dynamics by Arp2/3 and the autism and schizophrenia-associated protein Cyfip1. bioRxiv https://doi.org/10.1101/ 2020.05.31.124941 (2020).

59. Schaks, M. et al. Distinct interaction sites of Rac GTPase with WAVE regulatory complex have non-redundant functions in vivo. Curr. Biol. https://doi.org/ 10.1016/j.cub.2018.10.002 (2018).

60. Chen, B. et al. Rac1 GTPase activates the WAVE regulatory complex through two distinct binding sites. Elife https://doi.org/10.7554/eLife.29795. (2017).

61. Vivar, C. et al. Monosynaptic inputs to new neurons in the dentate gyrus. Nat. Commun. 3, 1107 (2012)

62. Tashiro, A. et al. NMDA-receptor-mediated, cell-specific integration of new neurons in adult dentate gyrus. Nature 442 https://doi.org/10.1038/ nature05028 (2006).

63. Bachmann, S. O. et al. Behavioral training rescues motor deficits in Cyfip1 haploinsufficiency mouse model of autism spectrum disorders. Transl. Psychiatry 9, 29 (2019).

64. Domínguez-lturza, N. et al. The autism- and schizophrenia-associated protein CYFIP1 regulates bilateral brain connectivity and behaviour. Nat. Commun. 10 3454 (2019)

65. Silva, A. I. et al. Cyfip1 haploinsufficient rats show white matter changes, myelin thinning, abnormal oligodendrocytes and behavioural inflexibility. Nat. Commun. 10, 3455 (2019).

66. Chaste, P. et al. Modest impact on risk for autism spectrum disorder of rare copy number variants at $15 q 11.2$, specifically breakpoints 1 to 2 . Autism Res. 7, 355-362 (2014).

67. Abdelmoity, A. T. et al. 15q11.2 proximal imbalances associated with a diverse array of neuropsychiatric disorders and mild dysmorphic features. J. Dev. Behav. Pediatr. 33, 570-576 (2012).

68. de Kovel, C. G. F. et al. Recurrent microdeletions at $15 q 11.2$ and 16 p13.11 predispose to idiopathic generalized epilepsies. Brain $\mathbf{1 3 3}$ 23-32 (2010).

69. Tay, T. L. et al. Microglia gone rogue: impacts on psychiatric disorders across the lifespan. Front. Mol. Neurosci. 10, 421 (2018).

70. Jones, K. A. \& Thomsen, C. The role of the innate immune system in psychiatric disorders. Mol. Cell. Neurosci. 53, 52-62 (2013). 